\title{
Adesão Terapêutica em Pacientes Diabéticos em Uso Medicamentoso
}

\author{
Emilly Bianca Oliveira Farias ${ }^{1}$; Marcelo José Costa Lima Espinheira ${ }^{2}$; \\ Nicio Roberto Sena Santana. ; Rafael Luiz de Araújo Rodrigues ${ }^{4}$
}

\begin{abstract}
Resumo: As doenças crônicas não transmissíveis (DCNTs), como o Diabetes Mellitus (DM), ocupam o primeiro lugar nas estatísticas globais de mortalidade: $73 \%$ das mortes em 2020 serão causadas por essas doenças. Como característica das DCNT, o tratamento do DM é complexo, pois depende diretamente da educação do paciente e de sua participação ativa no plano de cuidados para alcançar o controle glicêmico e prevenir complicações. Por meio do entendimento cotidiano dos participantes da equipe da Estratégia Saúde da Família, juntamente com o diagnóstico situacional de diversas regiões, o problema da baixa adesão está aumentando, portanto observa-se a necessidade de ampliar uma proposta de intervenção para transformar essa situação e, assim, obter resultados favoráveis no tratamento do Diabetes. A partir do estudo realizado, percebe-se que mais da metade dos pacientes entrevistados apresentou algum grau de dificuldade no grau de adesão ao consumo de medicamentos, o que poderia gerar diversas complicações. Portanto, é necessário planejar e implementar sistemas de educação continuada com os pacientes, visando esclarecer o estado de saúde de cada paciente, bem como a importância do tratamento realizado de forma adequada e contínua, além de permitir a conscientização dos profissionais de saúde. a promoção da autonomia, aceitação, conhecimento e adaptação do paciente em relação à sua própria doença.
\end{abstract}

Palavras Chave: Diabetes, Adesão Terapêutica, Educação Continuada.

\section{Therapeutic Adhesion in Diabetic Patients in Drug Use}

\begin{abstract}
Non-communicable chronic diseases (DCNTs), such as Diabetes Mellitus (DM), rank first in overall mortality statistics: $73 \%$ of deaths in 2020 will be caused by these diseases. As a characteristic of NCDs, DM treatment is complex because it depends directly on the education of the patient and their active participation in the care plan to achieve glycemic control and prevent complications. Through the daily understanding of the participants in the Family Health Strategy team, together with the situational diagnosis of several regions, the problem of low adherence is increasing, so there is a need to expand a proposal for intervention to transform this situation and, thus achieving favorable results in the treatment of Diabetes. From the study, it was observed that more than half of the patients interviewed presented some degree of difficulty in the degree of adherence to medication consumption, which could generate several complications. Therefore, it is necessary to plan and implement continuing education systems with the patients, aiming at clarifying the health status of each patient, as well as the importance of the treatment performed in an adequate and continuous way, as well as allowing the awareness of health professionals. the promotion of autonomy, acceptance, knowledge and adaptation of the patient in relation to their own disease.
\end{abstract}

Keywords: Diabetes, Therapeutic Adhesion, Continuing Education.

\footnotetext{
${ }^{1}$ Emilly Bianca Oliveira Farias. Discente do curso de Farmácia da Faculdade Independente do Nordeste - FAINOR, Vitória da ConquistaBahia. E-mail: milly.bianca@hotmail.com

2 Marcelo José Costa Lima Espinheira. Biólogo graduado pela Universidade Católica de Salvador - UCSAL, Pós graduado em Metodologia e Gestão do Ensino Superior pela Faculdade Independente do Nordeste - FAINOR, Meste em Religião/Educação na linha de pesquisa sobre Ética e gestão pela faculdade EST. Docente da FAINOR, FTC e UNINASSAU.

${ }^{3}$ Nicio Roberto Sena Santana. Discente do curso de Farmácia da Faculdade Independente do Nordeste - FAINOR, Vitória da Conquista-Bahia. E-mail: nicioroberto12@hotmail.com

${ }^{4}$ Rafael Luiz de Araújo Rodrigues. Mestrando em Saúde Coletiva da Universidade Federal da Bahia - UFBA. Especialista em Pericias Criminais e Toxicológica pela Faculdade das Aguas Emendadas - Brasília-DF e Professor e Coorientador da Faculdade Independente do Nordeste - FAINOR, Vitória da Conquista-Bahia. Email: faelfarmac@ @otmail.com
} 


\section{Introdução}

As doenças crônicas não transmissíveis (DCNT), como o Diabetes Mellitus (DM), aparecem entre as primeiras posições nas estatísticas de mortalidade mundial (73\%) dos óbitos até 2020 serão causados pelas DCNT. Aproximadamente 347 milhões de pessoas no mundo têm DM, sendo $90 \%$ do tipo 2. É estimado que em 2030 este número alcance 353 milhões de pessoas tornando-se a $7^{\mathrm{a}}$ principal causa de morte no mundo (Tavares, 2016).

O Diabetes Mellitus (DM) pode ser definido como uma síndrome de etiologia múltipla, devido à falta e/ou incapacidade da insulina em exercer adequadamente sua função. Entre seus vários tipos, o DM do tipo 2 se destaca, representando $90 \%$ dos casos, aparecendo geralmente na idade adulta, de acordo com Malta et al. (2017). As complicações podem ser agudas ou crônicas. Complicações agudas incluem hiperglicemia e hipoglicemia. As complicações crônicas podem ser complicações microvasculares, como retinopatia e nefropatia, e distúrbios neurológicos, como a neuropatia (Silva, 2008).

O tratamento da DM é complexo, pois depende direta e principalmente da educação do paciente com sua participação ativa no plano de cuidados para alcançar o sucesso, controle glicêmico e prevenir possíveis complicações Bennett (2014).

Diabetes Mellitus (DM) é um distúrbio metabólico crônico, caracterizado por níveis elevados de glicose sanguínea devido à deficiência de insulina, que pode ser classificada como tipo 1 (DM1) e tipo 2 (DM2). Pacientes com DM1 representam 10\% e são caracterizados por uma deficiência absoluta de insulina devido à destruição autoimune das células betapancreáticas que culmina com a interrupção da produção de insulina e conseqüentemente em um desequilíbrio metabólico grave, portanto, os portadores de DM1 são chamados de insulina dependente (Bezerra et al., 2014).

Esse processo pode levar meses ou anos, aparecendo clinicamente após a destruição de pelo menos $80 \%$ das células beta-pancreáticas. Na fase clínica, os sinais e sintomas anteriormente imperceptíveis ou ausentes começaram a manifestar-se de forma constante, como a poliúria (excesso de urina), polidipsia (sede excessiva), polifagia, astenia e perda de peso em Malta; Campos; et al. (2015).

A falta de adesão terapêutica não está associada apenas ao fato de ingerir ou não o medicamento prescrito, mas também de que maneira o paciente prossegue o tratamento. É necessário levar em consideração a participação e cooperação do paciente, bem como o seu comportamento, posicionamento frente a efeitos psicológicos relacionados à doença e sua convivência com a mesma (BEZERRA, et; al, 2014). 
Além do mais, outro motivo de grande importância que tem influenciado a não adesão e causado riscos à saúde do paciente é a utilização de forma abusiva de medicamentos sem prescrição medica, o que evidencia o uso irracional e automedicação, prejudicando por fim o tratamento Malta et al. (2018). Sendo assim, é essencial que sejam elaboradas políticas de expansão e inclusão de diretrizes na atenção básica que forneçam uma dimensão subjetiva dos usuários frente a esse problema de saúde, além do acompanhamento farmacoterapêutico (Guimarães, 2013).

A adesão terapêutica pode ser dividida em dois níveis. No primeiro nível, considerado elevado estão contidos os pacientes aderentes, aqueles pacientes que seguem totalmente o tratamento prescrito Malta; Stopa; et al. (2015). Em outro nível estão os pacientes desistentes, que são aqueles que abandonam o tratamento sem nenhuma explicação. Existem ainda os pacientes persistentes, que participam do grupo dos não aderentes, que são pacientes que chegam a comparecer às consultas, mas não seguem o tratamento (TAVARES, et; al 2015).

A adesão terapêutica sofre inúmeras influências que se caracterizam por fatores externos e também por fatores diretamente relacionados ao paciente Malta; Silva Jr (2014). Por isso se faz necessária à participação do farmacêutico, exercendo o acompanhamento farmacoterapêutico a fim de traçar intervenções que proporcionem qualidade de vida ao paciente e corroborem para o sucesso do tratamento (Bezerra, et, al 2014).

Através da compreensão diária dos participantes da equipe de Estratégia Saúde da Família, junto com o diagnóstico situacional de várias regiões o problema de baixa adesão terapêutica está em uma crescente. Assim observa-se que há necessidade de expandir uma proposta de intervenção para transformar essa situação, e assim obter resultados favoráveis no tratamento da Diabetes (Angelo, 2015).

Nesse contexto, este estudo objetivou identificar a adesão terapêutica medicamentosa em pacientes portadores de diabetes de uma Unidade de Saúde da Família.

\section{Metodologia}

Trata-se de uma pesquisa quantitativa do tipo descritiva e exploratória realizada numa Unidade de Saúde da Família situada em um município do Sudoeste Baiano. Participaram do estudo pacientes portadores de diabetes, com idade entre 20 a 70 anos. Para levantamento da população de estudo foram assinalados os diabéticos cadastrados e acompanhados pela unidade de saúde a partir das fichas de aprazamentos, que são utilizadas para o acompanhamento e 
agendamento de consultas. Com essas informações foi obtida uma quantidade de pacientes diabéticos na unidade, gerando um desvio padrão relacionado à confiança e prevalência.

Foi aplicado um questionário elaborado conforme as necessidades do estudo pelos autores da pesquisa contendo questões relacionadas a se o paciente tem dificuldade de acesso à unidade ou a medicamentos na unidade de saúde; se faz uso de medicamentos não prescritos pelo médico; e se esquece de tomar ou se suspendeu o uso do medicamento sem informar o médico.

O mecanismo para coleta de dados foi à aplicação de um questionário adaptado e adequado, às necessidades deste estudo, conforme metodologia proposta por Minayo. Esses dados obtidos foram analisados, correlacionados e comparados com intuito de obter o perfil da prevalência, conforme as variáveis: sexo, masculino e feminino; cor/raça; escolaridade; trabalho remunerado; acesso a Unidade de Saúde; dificuldade de acesso aos medicamentos; esquecimento, orientação médica e diminuição da dose.

\section{Resultados e Discussão}

O levantamento dos dados e fatores que interferem na adesão do paciente ao seu tratamento inclui diversos fatores, de acordo com o estudo realizado. Entre eles estão os aspectos como renda familiar, nível de escolaridade, dificuldade de se lembrar dos horários, interrupção no tratamento e falta de conhecimento sobre a doença e acesso a medicamentos utilizados, tal condição permite que o paciente não consiga alcançar o objetivo esperado e não tenha sucesso no tratamento.

Resultados mostraram que a maioria dos pacientes diabéticos era do sexo feminino (67\%). A maioria das mulheres se mostrou mais aderente ao tratamento e apresentou menor número de faltas às consultas médicas, mostrando que mulheres se preocupam mais com a saúde do que os homens. Dados estes de acordo com resultados encontrados em estudo de Busnelo (2001) sobre investigação da adesão terapêutica na população portuguesa, na qual a maior incidência dos pacientes com aderência terapêutica correspondia ao gênero feminino $(68,50 \%)$.

Segundo Busnelo et. al (2001), o risco de baixa adesão ao tratamento em pacientes jovens é maior em comparação aos idosos. Quanto mais avançada é a idade, mais os pacientes se sentem ameaçados pela doença. Pacientes jovens dispõem de menor tempo para aguardar atendimento ou até mesmo estão em fase assintomática da doença dificultando o tratamento. 
Tabela 1. Idade x Perguntas

\begin{tabular}{|c|c|c|c|c|c|}
\hline & & $\begin{array}{l}45 \text { a } 59 \\
\mathrm{n}(\%)\end{array}$ & $\begin{array}{l}60 \text { a } 69 \\
\mathrm{n}(\%)\end{array}$ & $\begin{array}{l}70 \\
\mathrm{n}(\%) \\
\end{array}$ & $\begin{array}{l}\mathrm{p}- \\
\text { valor }\end{array}$ \\
\hline \multirow{2}{*}{ Esqueceu medicamento Diabetes } & Sim & $42(72,4)$ & $25(75,8)$ & $6(60)$ & \multirow{2}{*}{0,666} \\
\hline & Não & $16(27,6)$ & $8(24,2)$ & $4(40)$ & \\
\hline \multirow{2}{*}{ Parou tomar sem avisar medico } & Sim & $30(51,7)$ & $22(66,7)$ & $6(60)$ & \multirow{2}{*}{0,286} \\
\hline & Não & $28(48,3)$ & $11(33,3)$ & $4(40)$ & \\
\hline \multirow{2}{*}{ Leva medicamento quando viaja } & Sim & $9(15,5)$ & $7(21,2)$ & $1(10)$ & \multirow{2}{*}{0,975} \\
\hline & Não & $49(84,5)$ & $26(78,8)$ & $9(90)$ & \\
\hline \multirow{2}{*}{ Parar tomar medicamento } & $\operatorname{Sim}$ & $28(48,3)$ & $14(42,4)$ & $3(30)$ & \multirow{2}{*}{0,282} \\
\hline & Não & $30(51,7)$ & $19(57,6)$ & $7(70)$ & \\
\hline \multirow{3}{*}{ Desistir tratamento } & Sim & $6(10,3)$ & $1(3)$ & $0(0)$ & \multirow{3}{*}{0,082} \\
\hline & Não & $50(86,2)$ & $30(90,9)$ & $9(90)$ & \\
\hline & Talvez & $2(3,4)$ & $2(6,1)$ & $1(10)$ & \\
\hline
\end{tabular}

Fonte: Pesquisa Própria

Com relação ao grau de escolaridade, os dados mostram que $54 \%$ dos pacientes não possuem escolaridade ou possuem baixa escolaridade, $43 \%$ não completaram o ensino fundamental e 3\% não chegou a completar o ensino médio.

A renda dos pacientes predominou a 01 salário mínimo, em torno de $\mathrm{R} \$ 980,00$ (51\%) apontando que a maioria dos pacientes eram aposentados. $\mathrm{O}$ descontrole na diabetes e sua não adesão apresentaram em maior frequência em pacientes que não possuía nenhum tipo de renda (49\%), pois o poder aquisitivo está relacionado com menor acesso ao medicamento, quando este não o possui na Unidade de Saúde e não tem alimentação adequada. (Mascarenhas et al., 2006).

Com relação ao conhecimento sobre a doença e os medicamentos utilizados, os resultados foram preocupantes, 58\% dos pacientes não possuía nenhum conhecimento ou conhecimento mínimo da Diabetes, podendo estar associado à baixa escolaridade ou então à falta de comunicação entre os profissionais da equipe de saúde (médico, enfermeiro, farmacêutico) e o paciente.

Jardim (2001) questionou que os conhecimentos do paciente sobre a doença e os medicamentos que utiliza são de grande importância, visto que eleva o comprometimento no autocuidado e adesão. Porém, a pesquisa realizada por Aquino et al., (2001), cita que o problema da adesão terapêutica é muito complexa, já que nem sempre fornecer acesso a informação sobre gravidade da doenças e suas complicações irão garantir a maior adesão às medidas de controle. 
Os autores Busnello et al.(2001), Lessa (1997) e Mascarenhas et al. (2006), apontam que a cronicidade da doença e a falta de sintomas explícitos são fatos importantes e relevantes para a interrupção do tratamento. No presente estudo foi possível identificar que $50 \%$ dos pacientes já interromperam o tratamento, pois sentiram que a glicemia estava controlada, alegando que não possuía nenhuma necessidade da administração do medicamento.

Tabela 2. Questionamento sobre a Adesão Terapêutica

\begin{tabular}{|c|c|c|c|c|}
\hline & & $\begin{array}{l}\text { Masculino } \\
\mathrm{n}(\%)\end{array}$ & $\begin{array}{l}\text { Feminino } \\
\mathrm{n}(\%)\end{array}$ & p-valor \\
\hline \multirow{2}{*}{ Esqueceu medicamento Diabetes } & Sim & $36(72)$ & $36(72)$ & \multirow{2}{*}{1,000} \\
\hline & Não & $14(28)$ & $14(28)$ & \\
\hline \multirow{2}{*}{ Parou tomar sem avisar medico } & Sim & $28(56)$ & $29(58)$ & \multirow{2}{*}{0,840} \\
\hline & Não & $22(44)$ & $21(42)$ & \\
\hline \multirow{2}{*}{ Leva medicamento quando viaja } & Sim & $5(10)$ & $11(22)$ & \multirow{2}{*}{0,103} \\
\hline & Não & $45(90)$ & $39(78)$ & \\
\hline \multirow{2}{*}{ Parar tomar medicamento } & Sim & $23(46)$ & $21(42)$ & \multirow{2}{*}{0,688} \\
\hline & Não & $27(54)$ & $29(58)$ & \\
\hline \multirow{3}{*}{ Desistir tratamento } & Sim & $7(14)$ & $0(0)$ & \multirow{3}{*}{0,565} \\
\hline & Não & $38(76)$ & $50(100)$ & \\
\hline & Talvez & $5(10)$ & $0(0)$ & \\
\hline
\end{tabular}

Fonte: Pesquisa Própria

Em relação ao tratamento farmacológico $65 \%$ dos pacientes mostraram dificuldade de lembrar-se de tomar os medicamentos e $45 \%$ não se lembravam dos horários corretos de cada medicamento. Ao sair para alguma viagem ou grande tempo fora de casa, 78\% das mulheres afirmaram levar seus medicamentos, enquanto $90 \%$ dos homens não levavam. 55\% apresentavam dificuldade em adesão ao tratamento, ou seja, em seguir o esquema terapêutico, pois segundo Osterberg \& Blaschke, 2005; Coelho \& Nobre, 2006 quanto mais simples for o esquema, maior é a adesão ao tratamento facilitando o entendimento e consequentemente o sucesso do tratamento.

Segundo $62 \%$ dos pacientes masculinos e femininos abordados houve interrupção do tratamento por um tempo ( $58 \%$ público feminino e $56 \%$ masculino), informaram ser por causa da ausência de sintomas, por sentir efeitos colaterais, falta de medicamentos na Unidade, dificuldade no agendamento de consultas, relacionamento com os profissionais de saúde na unidade, e por falta de tempo, se equiparando com estudos realizados por Mascarenhas et al., (2006) na Bahia. 
Aspectos de atenção à saúde, como bom relacionamento com os profissionais de saúde, devem ser revisados e aprimorados, pois são aspectos importantes para a adesão do paciente. Tais ações incentivam os pacientes a cuidar de sua saúde, minimizar as barreiras que muitas vezes impedem o acesso à Unidade e facilitam o acesso aos medicamentos. (Tavares et al., 2016).

Lessa (2006) mostrou que é extremamente necessário que os pacientes conheçam sua doença, seus medicamentos e sua dosagem. Para isso, é importante que as informações repassadas aos pacientes sejam por meio de profissionais de saúde treinados e que as orientações sejam claras e concisas, relacionadas não apenas às expectativas da doença, mas também esclarecimentos relacionados aos medos e tabus dos pacientes, bem como para o tratamento como um todo e suas restrições.

\section{Conclusões}

É necessário planejar e implementar sistemas de educação continuada com os pacientes, visando esclarecer a condição de saúde de cada um, bem como a importância do tratamento realizado de forma adequada e contínua. Além disso, permitir que os profissionais de saúde conscientizem sobre a autonomia, aceitação, conhecimento e adaptação do paciente em relação à sua própria doença.

Aspecto referente ao serviço de saúde como o bom relacionamento com os profissionais de saúde deve ser revisto e aprimorado, pois este é um aspecto importante para a adesão dos pacientes, denominando-os para cuidar de sua saúde minimizando as barreiras que os impedem de frequentar a Unidade periodicamente.

\section{Referências}

BENNETT, D. M. Fatores de risco associados às doenças crônicas não transmissíveis: uma revisão bibliográfica. British Journal of Psychiatry, v. 205, n. 01, p. 76-77, 2014.

BEZERRA, A.S.; LOPES, J.L.; BARROS, A.L Adesão de Pacientes Hipertensos ao Tratamento Medicamentoso. Rev Bras Enferm, v. 67, n. 4, p. 550-5, 2014.

BRASIL. Ministério da Saúde. Secretaria de Ciência, Tecnologia e Insumos Estratégicos. Departamento de Ciência e Tecnologia. Síntese de evidências para polítcas de saúde: adesão 
ao tratamento medicamentoso por pacientes portadores de doenças crônicas. Brasília: Ministério da Saúde, 2016.

GIROTTO, E; MAFFEI, S; SARRIA, A. Adesão ao tratamento farmacológico e não farmacológico e fatores associados na atenção primária da hipertensão arterial, Ciência \& Saúde Coletiva, v.18, n.6, p.1763-1772, 2013.

GUIMARÃES, A. C. Uso e abuso dos benzodiazepínicos: revisão bibliográfica para os profissionais de saúde da Atenção Básica, Curso de especialização em Atenção Básica em Saúde da Família. Trabalho de Conclusão de Curso (Especialização em Atenção Básica em Saúde da Família) - Universidade Federal de Minas Gerais, Belo Horizonte - MG, 2013.

MALTA, D. C.; BERNAL, R. T. I.; LIMA, GUIMARÃES, M.; et al. Doenças crônicas não transmissíveis e a utilização de serviços de saúde: análise da Pesquisa Nacional de Saúde no Brasil. Rev Saude Publica, v. 51, n. 1, p. 1s-10s, 2017.

MALTA, D. C.; CAMPOS, M. O.; OLIVEIRA, M. M. DE; et al. Prevalência de fatores de risco e proteção para doenças crônicas não transmissíveis em adultos residentes em capitais brasileiras, 2013. Epidemiologia e Serviços de Saúde, v. 24, n. 3, p. 387-373, 2015.

MALTA, D. C.; DUNCAN, B. B.; BARROS, M. B. DE A.; et al. Medidas de austeridade fiscal comprometem metas de controle de doenças não transmissíveis no Brasil. Ciência \& Saúde Coletiva, v. 23, n. 10, p. 3115-3122, 2018.

MALTA, D. C.; SILVA JR, J. B. DA. Plano de Ações Estratégicas para o Enfrentamento das Doenças Crônicas Não Transmissíveis no Brasil após três anos de implantação, 2011-2013. Epidemiologia e Serviços de Saúde, v. 23, n. 3, p. 389-398, 2014.

MALTA, D. C.; STOPA, S. R.; SZWARCWALD, C. L.; et al. A vigilância e o monitoramento das principais doenças crônicas não transmissíveis no Brasil - Pesquisa Nacional de Saúde, 2013. Revista Brasileira de Epidemiologia, v. 18, n. suppl 2, p. 3-16, 2015.

MUNIZ L. A. Adesão ao tratamento e controle da Hipertensão Arterial - Proposta de Intervenção. Trabalho de Conclusão de Curso (Especialização em Atenção Básica em Saúde da Família) - Universidade Federal do Triângulo Uberaba - MG, 2015.

NAVARRO, A.; NAVARRO, F. Perfil dos Fatores de Risco para Doenças Crônicas NãoTransmissíveis em Escolares da Zona Urbana e Rural. Revista Brasileira de Prescrição e Fisiologia do Exercício, v. 12, n. 72, p. 38-45, 2013.

SILVA, N. R.; COSTA, C. E. M. A hiperglicemia e os mecanismos envolvidos nas disfunções vasculares do Diabetes Mellitus. Arq. Ciênc. Saúde Unipar, Umuarama, v. 12, n. 3, p. 265 270, set./dez. 2008.

TAVARES, D.M.S; GUIMARAES, M,O; FERREIRA, P,C,S; MARTINS, F, N, P. Qualidade de vida e adesão ao tratamento farmacológico entre idosos hipertensos, Rev Bras Enfermagem v.69, n.1, p.134-41, jan-fev 2016. 
TAVARES, N. U. L.; COSTA, K. S.; MENGUE, S. S.; et al. Uso de medicamentos para tratamento de doenças crônicas não transmissíveis no Brasil: resultados da Pesquisa Nacional de Saúde, 2013. Epidemiologia e Serviços de Saúde, v. 24, n. 2, p. 315-323, 2015.

TAVARES, N; BERTOLDI, A. Fatores associados à baixa adesão ao tratamento farmacológico de doenças crônicas no Brasil, Rev Saúde Pública, v.50, (supl 2), p. 11s, 2016.

\section{Como citar este artigo (Formato ABNT):}

FARIAS, Emilly Bianca Oliveira; ESPINHEIRA, Marcelo José Costa Lima; SANTANA, Nicio Roberto Sena; RODRIGUES, Rafael Luiz de Araújo. Adesão Terapêutica em Pacientes Diabéticos em Uso Medicamentoso. Id on Line Rev.Mult. Psic., 2019, vol.13, n.43, p. 407-415. ISSN: 1981-1179.

Recebido: 29/11/2018;

Aceito: 30/11/2018 